\title{
The endogenous apolipoprotein A-I enhances the uptake of oxidated Low Density Lipoprotein by macrophages
}

E. Nekrasova, A. Nikitin, D. Mogilenko, V. Shavva, V. Kudriavtsev, S. Orlov Institute of Experimental Medicine, department of Biochemistry, 197376, acad. Pavlov st., 12, Saint-Petersburg, Russia. E-mail: serge@iem.sp.ru, Phone: +79213272095

\section{Introduction}

Apolipoprotein A-I (ApoA-I) is the main structural and functional protein component of high-density lipoprotein. Recently we found the moderate expression of endogenous apoA-I in human monocytes and macrophages and showed the antiinflammatory activity of endogenous ApoA-I in macrophages. The homogeneous population of monocytes divides to the ApoAI-rich and ApoA-I-poor macrophages during the differentiation. The functions of macrophagal ApoA-I in the development of atherosclerotic lesions are still unknown. Here we show the participation of macrophagal ApoA-I in the uptake of oxidized Low Density Lipoprotein (oxLDL) and study the ApoA-I gene regulation in macrophages by oxLDL.

The oxLDL uptake is by ApoA-I rich macrophages. Confocal microscopy mLDL ApoA-I DAPI
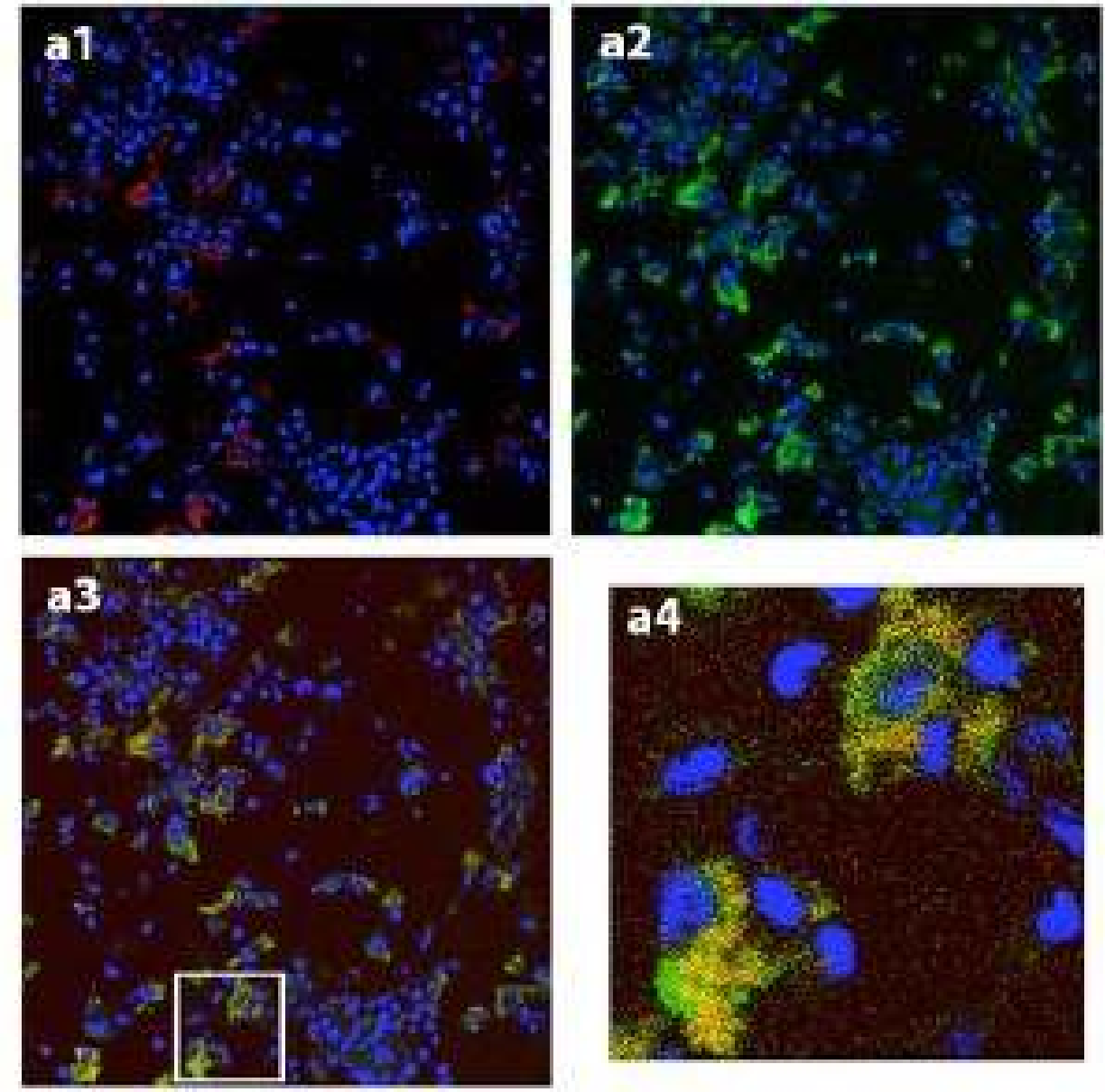

The knockdown ApoA-I, ABCA-1 leads to a decrease scavenger receptor LOX-1

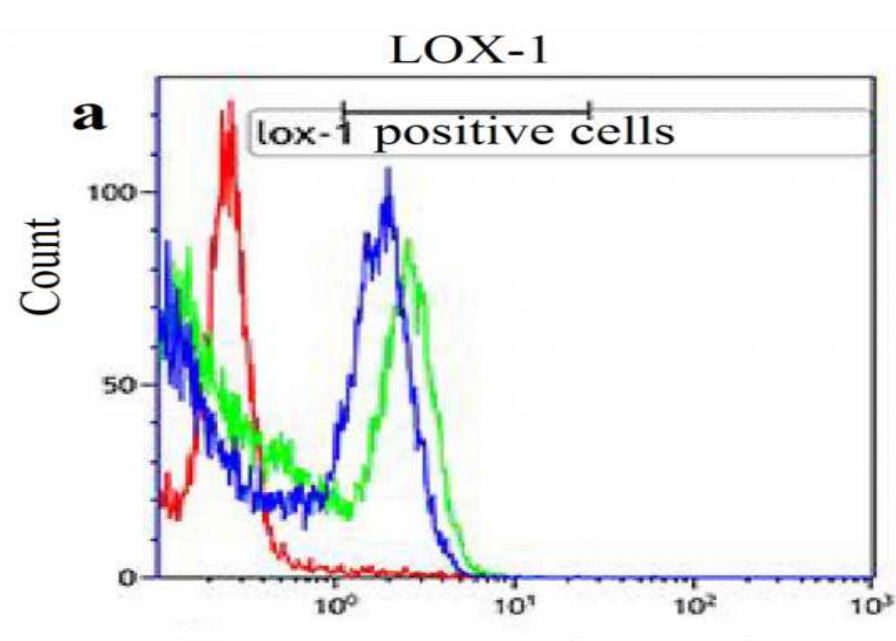

$\square$ siApoA-I
$\square$ scrambled RNA

Fluorescence intensity

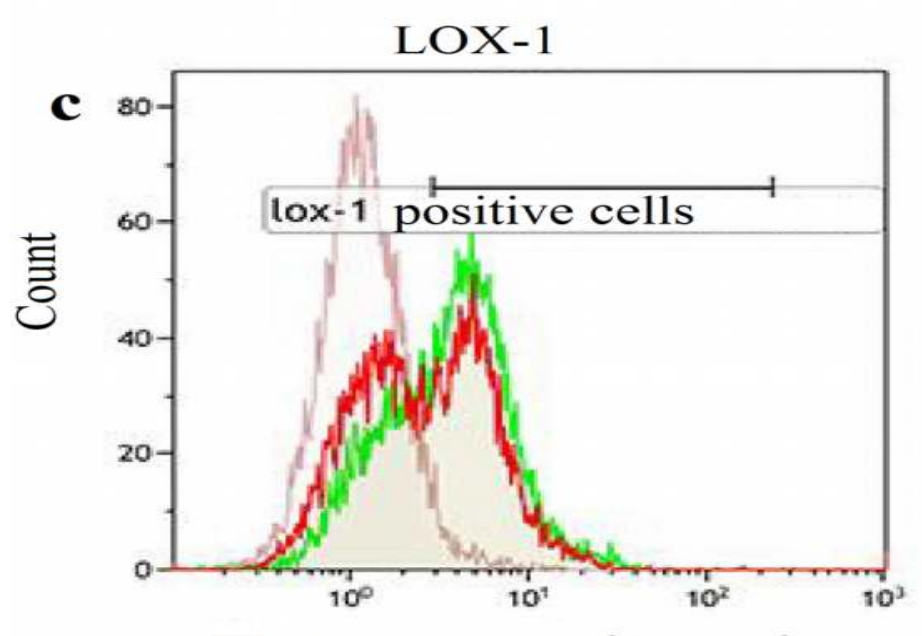

Fluorescence intensity

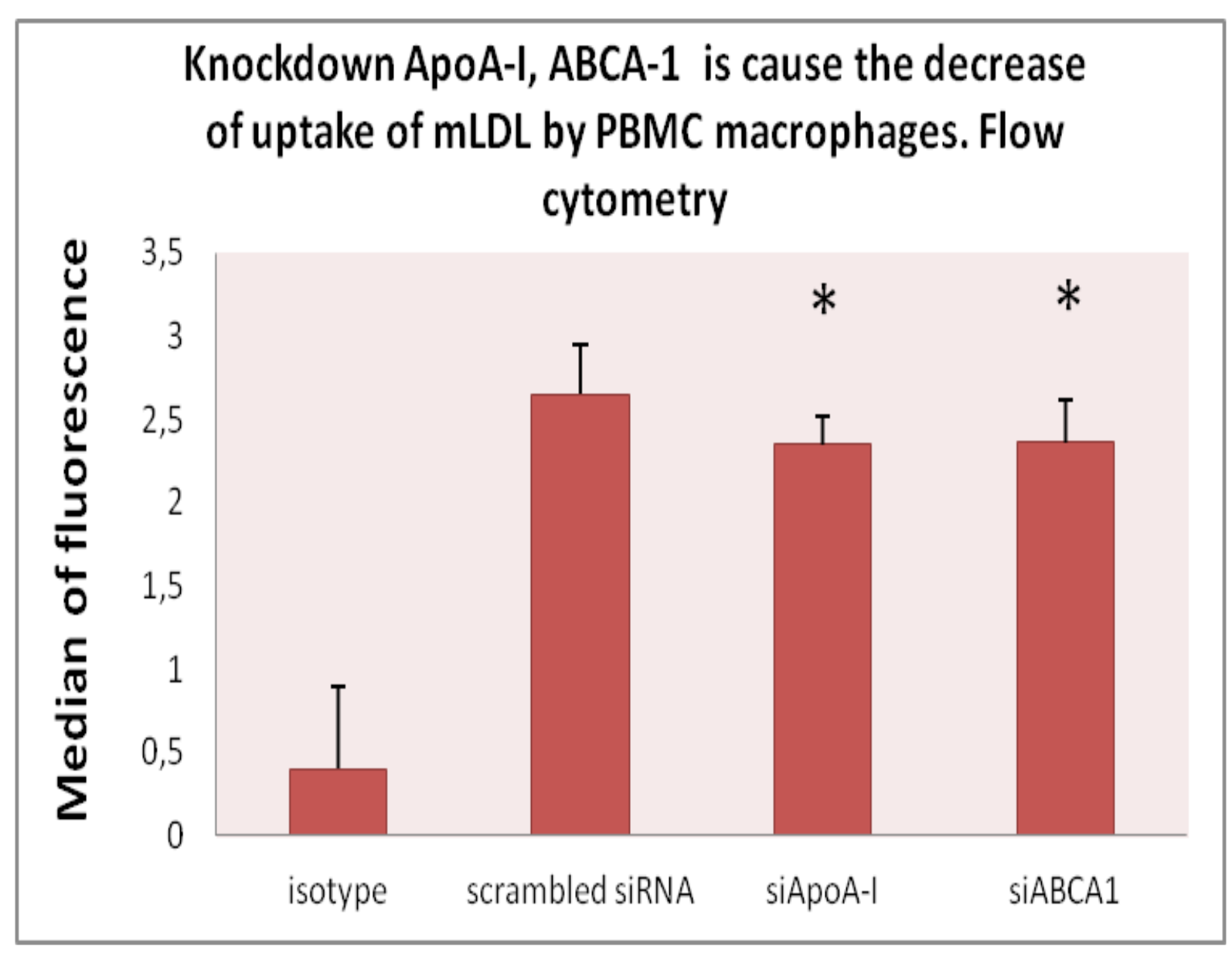

The role of signaling cascades in ApoA-I mediated upregulation LOX-1 gene 450 in human macrophages. RT-PCR.

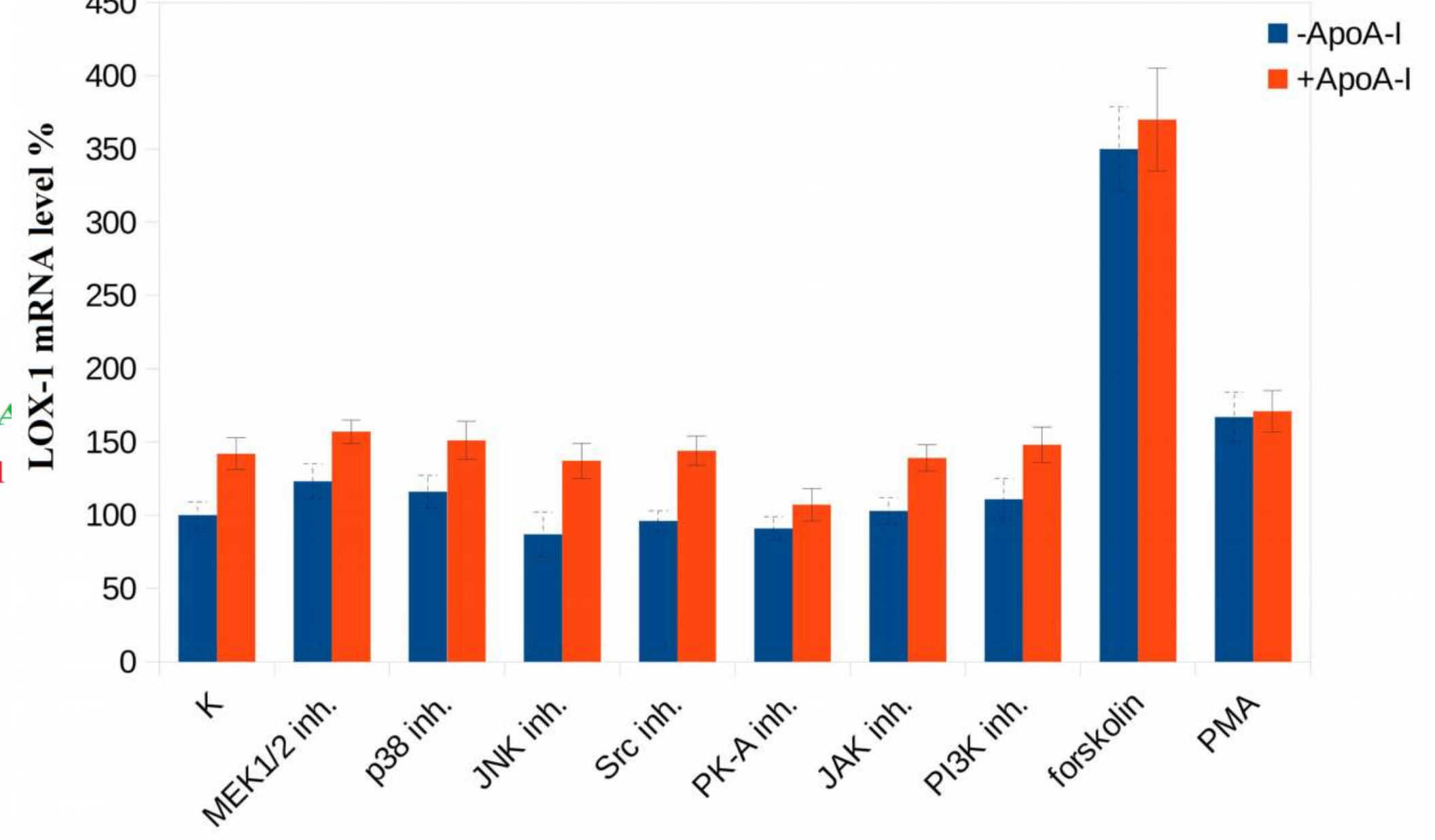

\section{Conclusions}

The endogenous ApoA-I in macrophages enhances oxLDL uptake through the induction of scavenger receptor LOX1 gene. 